\title{
Die „Erneuerung der Verwaltung“ in Japan
}

\author{
Von Atsushi Takada, Osaka*
}

\section{A. Einleitung}

I. Drei Neuerungen im japanischen Verwaltungsrecht

In diesem Beitrag werden Erneuerungen des Verwaltungsrechts in Japan behandelt. Zu den wichtigen Erneuerungen können folgende drei Erscheinungen gezählt werden:

\section{Organisatorische „Reformen“}

Als wichtigste Erscheinungen können organisatorische „Reformen“, die durch die sog „Reform der Zentralregierung“ seit den 1990er Jahren betrieben wurde, angesehen werden. Diese „Reformen“ haben auch nach dem Regierungswechsel von der LDP zu den Demokraten im Jahr 2009 eine bemerkenswerte Fortsetzung gefunden und weisen deutliche Symptome als Misserfolg auf.

\section{2. „Transparenz“ als neuer materieller Grundsatz}

Auch die Steigerung der „Transparenz“ ist eine wichtige Erneuerung. Schon durch Verabschiedung des Verwaltungsverfahrensgesetzes (1993) und des Gesetzes zum Zugang zu Verwaltungsinformationen (1999) begann die Entwicklung in diese Richtung. Zur weiteren Steigerung wurde ein weiteres wichtiges System eingeführt, nämlich die „Bewertung“ der Verwaltungstätigkeiten, die auch kritische Beobachtungen erfordert.

\section{Neuerungen in der Verwaltungsprozessordnung}

Im Jahr 2004 wurden an der Verwaltungsprozessordnung wichtige Änderungen für den effektiven Rechtsschutz, Änderungen für die Erweiterung des Umfangs des Rechtsschutzes und die Förderung der Benutzung des Verwaltungsprozesses vorgenommen. Es wird erwartet, dass diese Änderungen langfristig für den Rechtsschutz sowie die Verwaltungskontrolle durch Gerichte positive Wirkungen haben werden. Es ist aber noch zu früh, Wirkungen dieser Änderungen wissenschaftlich zu kommentieren.

In diesem Beitrag werden daher die unter 1. und 2. angeführten Änderungen, die sich seit 15 Jahren gemeinsam entwickeln ${ }^{1}$, im Überblick vorgestellt und analysiert.

* Atsushi Takada, Dr. jur., Studium an der Universität Kyoto und an der Universität Köln. Professor für Öffentliches Recht an der Universität Osaka. Der vorliegende, im Rahmen des von der Alexander von Humboldt Stiftung geförderten Forschungsaufenthalts an der Freien Universität Berlin verfasste Beitrag ist eine überarbeitete Fassung des Vortrages, den der Verfasser auf der Jahrestagung des Arbeitskreises für Überseeische Verfassungsvergleichung am 3. Juli 2010 in Regensburg gehalten hat. 


\section{Universalität und Besonderheiten der „Reformen“}

Es kann universal beobachtet werden, dass sich um „Reformen“ bemüht wird, um die Verwaltungsorganisationen zu ändern und Verwaltungstätigkeiten effizienter sowie transparenter zu machen. „Reformen“ in Japan können als Teil dieser universalen Erscheinungen betrachtet werden. Japanische Erneuerungen haben aber ihre Besonderheiten, die aus dem japanischen Kontext folgen. Es ist daher notwendig, Universalität und Besonderheiten der Erneuerungen der Verwaltung in Japan wissenschaftlich zu beobachten und damit einen Beitrag zur Rechtsvergleichung zu versuchen.

\section{Methodische Erwägungen}

1. „Reformen“ der Verwaltung als gesellschaftliche Phänomene

Die „Reformen“ der Verwaltung in Japan sind gesellschaftliche Phänomene, die in gesellschaftlichen Zusammenhängen in Japan stehen. Um sie wissenschaftlich zu analysieren, wird eine geeignete Methode benötigt. In diesem Beitrag wird versucht, die systemtheoretische Methode anzuwenden.

\section{Erneuerungen des Verwaltungsrechts als normative Phänomene}

Die Erneuerungen des Verwaltungsrechts sind gleichzeitig rechtliche Phänomene, mit denen Juristen meistens dogmatisch und rechtstheoretisch umgehen. Wenn ein Jurist einen bestimmten Überblick zum Öffentlichen Recht in Japan hat, gibt es kein Problem, dogmatische und rechtstheoretische Probleme zu verstehen. Es ist jedoch sehr schwierig, einen Überblick in diesem kurzen Beitrag zu schaffen. Ich werde daher versuchen die normative Logik, die hinter den Erneuerungen steht zu ermitteln, sie vorzustellen und zu analysieren.

\section{B. Die ,Verwaltungsreform“ in den 1990er Jahren}

Seit der Entstehung der „Japanischen Verfassung“ (1946, =JV) wurde eine „Verwaltungsreform“ ständig diskutiert. In den 1980ern wurde sogar die Rollenverteilung zwischen öffentlichem und privatem Sektor thematisiert, woraufhin das Eisenbahnwesen und Telekommunikationswesen in der Tat privatisiert wurden. Von diesen Reformdiskusionen und „Reformen“ sollte die „Verwaltungsreform“ in den 1990er Jahren aber grundsätzlich unterschieden werden: Sie kann dadurch charakterisiert werden, dass es um die umfassende „Reform“ der Staatsstruktur ging, obwohl auch die Rollenverteilung zwischen öffentlichem und privatem Sektor thematisiert wurde. 


\section{Der Hintergrund der grundsätzlichen „Verwaltungsreform“}

Der wichtigste Erklärungsfaktor für die neuen Bemühungen zur grundsätzlichen „Verwaltungsreform“ ist, dass die Verwaltung in den 1990er Jahren plötzlich das Vertrauen der Bevölkerung verloren hatte. In Bezug auf die wirtschaftliche Krise in diesem Jahrzehnt konnten das MITI (vom engl. Ministry of International Trade and Industry) und das Finanzministerium keine effektiven Maßnahmen ergreifen, schlimmer noch, letztere Behörde wirkte sogar als Hindernis auf dem Weg zur Erholung der Konjunktur. Bei der Erdbebenkatastrophe in Kobe entstand darüber hinaus bei der Bevölkerung der Eindruck, dass der „Staat“ in Krisensituationen der Bevölkerung nicht helfen kann. Auch der „AIDSSkandal“ zeigte Schwachstellen des Systems auf. Es wurde deutlich, dass die Gesundheitspolitik in Japan nicht primär an der Gesundheit der Bevölkerung, sondern an der Stabilität der Pharmaindustrie orientiert war, die eine stabile Versorgung mit Arzneimitteln ermöglicht. Außerdem rückten zahlreiche Korruptionsfälle die Beamten selbst ins Blickfeld. Bis zu diesem Zeitpunkt hatte die Verwaltung eine sehr starke Position in der Staatsstruktur und ein hohes Ansehen in der Gesellschaft genossen. Die Staatsstruktur in Japan, die von der ,starken“ Verwaltung geprägt war, wurde nun aber von der Bevölkerung nicht mehr als zeitgemäß betrachtet.

Die Destabilisierung der Politik in den 1990er Jahren hat auch eine nicht unwesentliche Rolle bei der Durchsetzung der „Verwaltungsreform“ gespielt. Die politische Stabilität, für die der Fortbestand der absoluten Mehrheit der LDP charakteristisch war, existierte nicht mehr, obwohl die LDP weiter die relativ stärkste Partei blieb. Alle Parteien, auch die LDP, mussten versuchen, dadurch Stimmen der Wähler zu gewinnen, dass sie sich als „Reformer“ präsentierten. So brach die „Ära der Reform “" an. Als wichtigstes Reformthema wählte man dabei die „Verwaltungsreform“, die wegen der Übermacht der Verwaltung in Japan als „Reform der Reformen“ galt.

\section{II. „Konferenz der Verwaltungsreform“ als Konstrukteur der „Reform“}

Mit der Unterhauswahl im Jahr 1996, bei der die „Verwaltungsreform“ das wichtigste Wahlkampfthema war, wurde die LDP-Regierung von Premierminister Hashimoto bestätigt. Hashimoto gründete zur Verwirklichung des Wahlversprechens der „Verwaltungsreform“ die „Konferenz zur Verwaltungsreform“ aus Vertretern wichtiger gesellschaftlicher Kräfte und führender Fachleute und übernahm selbst das Amt des Vorsitzenden dieser Konferenz.

Die „Verwaltungsreform“ wurde aufgrund der Diskussionen und des Berichts der „Konferenz“ entworfen und umgesetzt. Die außerordentliche Einflusskraft der „Konferenz“

2

Zu wichtigen „Reformen“ in dieser Zeit zählen auch die „politische Reform“, die „Reform zur Zentralisierung“ und die „Justizreform“. Über die ,politische Reform“ siehe Atsushi Takada, Die Parteiinstitution in Japan, in: D. Tsatsos (Hrsg.), 30 Jahre Parteiengesetz in Deutschland Aspekte aus Wissenschaft und Politik, 2001, 306 ff. 
könnte teilweise dadurch zu erklären sein, dass die Tätigkeit der „Konferenz“ in hohem Maße öffentlich und transparent stattfand. Premierminister Hashimoto übernahm selbst das Amt des Vorsitzenden der Konferenz, weshalb der Bericht der „Konferenz“ unbedingt von der Regierung beachtet werden musste. Durch sein aktives Engagement konnte das Vorgehen der „Konferenz“ große Aufmerksamkeit der Öffentlichkeit auf sich ziehen. Protokolle und Materialien der „Konferenz“ wurden frühzeitig der Öffentlichkeit zugänglich gemacht, sogar über das Internet. Die bisher beispiellose Öffentlichkeit und Transparenz der „Konferenz" machte es der Verwaltung schwer, bei der Durchführung der Maßnahmen, die in dem Bericht der „Konferenz“ vorgeschlagen wurden, Widerstand zu leisten.

\section{Zentrale Reforminhalte}

1. Bericht der „Konferenz“: „Kokumin“ (Volk, Staatsbürger) als Subjekt staatlicher Herrschaft

Der Bericht der „Konferenz“ sah die umfassende Reform der Staatsstruktur durch konkrete Maßnahmen der „Verwaltungsreform“ vor. Es muss dabei betont werden, daß mit der „Reform“ neben der Umsetzung der konkreten Vorschläge die „Konstruktion dieses Landes (kono Kuni no Katachi)“ beabsichtigt war. Der Bericht lautet:

„Nach der Niederlage im 2. Weltkrieg hat dieses Land sich so konzentriert darum bemüht, alle gesellschaftlichen Kräfte zur wirtschaftlichen Konkurrenz zu mobilisieren, so dass Japan eine Wirtschaftsmacht werden konnte. Dieses Land wird aber aufgrunddessen von Einschränkungen und Konventionen beherrscht, mit dem Ergebnis einer sehr standardisierten und starren Gesellschaft. Diese Reform ist die Reform der Verwaltung, gleichzeitig auch die Reform der Konstruktion dieses Landes, in der das Volk sich an das Bewußtsein als Herrschaftsobjekt gewöhnt hat und meist von der Verwaltung abhängig ist. Diese Verwaltungsreform bezweckt, das ermüdete Verwaltungssystem nach dem 2. Weltkrieg zu reformieren, um ein Verwaltungssystem im 21. Jahrundert zu schaffen, das zur Verwirklichung der aufgrund von autonomen Individuen freien, gerechten Gesellschaft passt“. ${ }^{3}$

Der Bericht nimmt dabei Bezug auf die japanische Verfassung, die vorsieht, dass jeder Staatsbürger („Kokumin“ auf Japanisch) als Individuum respektiert wird. (Art. $13 \mathrm{JV}^{4}$ ), und dass die Souveränität dem Volk (,Kokumin“ auf Japanisch) zusteht (Präambel JV ${ }^{5}$ ).

Die Konferenz der Verwaltungsreform, Der Endbericht der Konferenz der Verwaltungsreform, 1997, S. 2 ff.

4

Art. 13 JV: Jeder Staatsbürger (Kokumin) wird als Individuum respektiert. Die Rechte des Volkes auf Leben, Freiheit und das Streben nach Wohlstand müssen, soweit sie nicht zum öffentlichen Wohl im Widerspruch stehen, in der Gesetzgebung und anderen Maßnahmen des Staates größte Beachtung finden.

Präambel JV: Wir, das japanische Volk (Kokumin), handeln durch unsere rechtmäßig gewählten Vertreter im Parlament und sind entschlossen, für uns und für unsere Nachkommen die Früchte friedlicher Zusammenarbeit mit allen Völkern und die Segnungen der Freiheit überall auf dem Boden unseres Staates zu sichern, auf dass nie wieder durch Handlungen der Regierung die 
2. „Vier Säulen“ als Grundbausteine der Reform

Für eine systematische Übersicht über die konkreten Vorschläge der „Konferenz“ ist es sinnvoll, sich diese „Reformen“ als aus vier Säulen bestehend vorzustellen.

a) Organisationsreform zur Stärkung der politischen Führung

Die erste Säule der „Reform“ war die organisatorische Umgestaltung mit dem Ziel der effektiven Führung. Dieser Aspekt steht auch im Bericht im Mittelpunkt. Der Bericht betrachtet diese Maßnahme als besonders notwendig, um eine konsequente strategische Urteils- und Entscheidungskraft der Regierung über die Verwaltung sicherzustellen(,,topdown“). Um die Überordnung der Regierung gegenüber der Verwaltung, also der Politik gegenüber der Bürokratie zu verwirklichen, musste eine effektive politische Führung durch den Premierminister, sein Kabinett sowie seine Staatsminister durchgesetzt werden.

Aufgrund der Befugnis des Kabinetts zur „Leitung der Staatsgeschäfte“ (Art. 73 Nr. 1 $\mathrm{JV}^{6}$ ) sowie der Position des Premierministers als „Oberhaupt“ des Kabinetts (Art. 66 Abs. $1 \mathrm{JV}^{7}$ ), wurde das Vorschlagrecht des Premierministers in Bezug auf ,politische Richtlinien des Kabinetts“ im Kabinettsgesetz neu bestimmt (Art. 4 Abs. 2). Für Plan- und Programmgestaltung sowie die Gesamtkoordination der Richtlinien ist nun das Kabinettssekretariat zuständig (geänderte Fassung des Art. 12 Abs. 2 KabinettsG). Zur Unterstützung dieser Plan- und Programmgestaltung sowie Gesamtkoordination wurde die Kabinettszentrale neu gegründet (Gründungsgesetz der Kabinettszentrale). In dieser Zentrale wurden vier „Konferenzen“ für wichtige politische Angelegenheiten (,Wirtschaft und Finanzen“ u.s.w.) geschaffen, die aus dem Premierminister, der grundsätzlich als Vorsitzender tätig ist, den betreffenden Staatsministern und vom Premierminister ernannten Sachverständigen bestehen (Grundungsgesetz der Kabinettszentrale Art. 18).

Ebenfalls zur Stärkung der politischen Führung wurden sowohl im Kabinettssekretariat als auch in jedem Ministerium neue politische Ämter eingeführt. In jedem Ministerium wurde z.B. ein Vizeminister neu besetzt und die Zahl der politischen Sekretäre des Ministers vergrößert.

Greuel eines Krieges entstehen können, verkünden hiermit, dass die Souveränität dem Volk (Kokumin) zugehört, und beschließen diese Verfassung. Denn eine Regierung beruht auf dem ernsthaften Vertrauen des Volkes, ihre Autorität geht vom Volk aus, ihre Macht üben Vertreter des Volkes aus, und ihre gesegneten Leistungen genießt das Volk. Das ist ein universell gültiges Prinzip der Menschheit, und diese Verfassung beruht auf diesem Prinzip. Wir setzen ohne Ausnahme Verfassungen, Gesetze, Verordnungen und Erlasse, die im Gegensatz hierzu stehen, außer Kraft.

Art. 73 JV: Außer allgemeinen Verwaltungsaufgaben obliegen dem Kabinett folgende Geschäfte: 1. Die gewissenhafte Durchführung der Gesetze und die Leitung der Staatsgeschäfte...

7 Art. 66: (1) Das Kabinett besteht aus dem Premierminister als Oberhaupt und den übrigen Staatsministern, wie es im Gesetz vorgesehen ist. 
b) Restrukturierung der Ministerien

Die Zweite Säule der Reform bestand aus der Restrukturierung der nationalen Verwaltungsorgane, eine Änderung, die im Bericht der „Konferenz“ für die Konsequenz, Zweckrationalität und Effektivität in der Verwaltung als notwendig erachtet wurde. Die ,verschiedenen Zweige der Verwaltung“ (Art. $72 \mathrm{JV}^{8}$ ) besaßen bisher bei geringerer Kontrolle von oben oder außen große Spielräume, auf deren Grundlage sie ihre Aktivitäten ganz frei in verschiedene Richtungen entwickeln konnten, woraus manchmal Ineffizienzen, Überschneidungen und Widersprüche zwischen den ,verschiedenen Zweigen“ resultierten.

Um die hier erwähnten großen Spielräume der einzelnen Behörden, deren Ausschöpfung manchmal regelrecht in Willkür des Verwaltungshandelns mündete, zu begrenzen, wurden die Bestimmungen über die Befugnisse jedes Ministeriums, die im jeweiligen Gründungsgesetz normiert waren und wegen ihres allgemeinen Inhalts dem Ministerium eine umfassende Rechtsgrundlage für seine Tätigkeiten gaben, abgeschafft. Um effizienzmindernde Kompetenzüberschneidungen und Widersprüche zwischen den Ministerien zu vermeiden, wurden die vormals existierende Kabinettszentrale und die ebenfalls in dieser Form nicht mehr existenten 22 Ministerien zu einer Zentrale und 12 Ministerien neu geordnet.

c) Transparenz als Grundzug moderner bürgernaher Verwaltung

Die dritte Säule der „Reform“ zielte auf die Steigerung der Transparenz der Verwaltung. Durch die Verabschiedung des Verwaltungsverfahrensgesetzes (1993) und des Gesetzes zum Zugang zu Verwaltungsinformationen (1999) begann eine Entwicklung in diese Richtung schon vor der „Reform“. Durch die „Reform“ wurde in diesem Zusammenhang jedoch ein weiteres wichtiges Element eingeführt, nämlich die Bewertung der Verwaltungstätigkeit.

d) Personal- und Sachmittelabbau

Die vierte und letzte Säule der „Reform“ war die drastische Verkleinerung der Verwaltung, die im Bericht der „Konferenz“ als unbedingt notwendig für die Erhöhung der Effektivität erachtet wurde.

Bei der Restrukturierung der nationalen Verwaltungsorgane wurde nicht nur die Zahl der Ministerien reduziert, sondern auch die Zahl der ihnen untergeordneten Verwaltungseinheiten.

89 Verwaltungsorganisationen, wie z.B. nationale Museen oder Forschungsinstitute, die der Aufsicht verschiedener Ministerien untergeordnet waren, wurden durch die

8

Art. 72 JV: Der Premierminister bringt als Vertreter des Kabinetts beim Parlament Vorlagen ein, er macht dem Parlament Mitteilung von den allgemeinen Staatsgeschäften und von den Beziehungen zum Ausland, er leitet und beaufsichtigt darüber hinaus die verschiedenen Zweige der Verwaltung. 
„Reform“ in selbständige Verwaltungsorganisationen umgewandelt. Ihre Leistungen werden von unabhängigen dritten Organen, bestehend aus Sachverständigen, bewertet.

Die Anzahl der öffentlichen Bediensteten wurde ebenfalls reduziert. Die gesetzlich bestimmte Personalstärke der verschiedenen Verwaltungsinstitutionen wurde planmäßig um 10 Prozent reduziert. Durch Privatisierung oder Einführung der weiteren selbständigen Verwaltungsorganisationen sollte eine Reduzierung um bis zu 25 Prozent erreicht werden.

\section{Weitere Entwicklung}

I. $\quad$ Erfolge und Misserfolge der Stärkung der politischen Führung

1. Erfolge

Die starke politische Führung wurde in der Regierung von Premierminister Koizumi (von 2001 bis 2006) mehrfach deutlich. Aufgrund der Organisationsreform zur Stärkung der politischen Führung und der politischen Zuspitzungsfähigkeit von Koizumi konnten einige wichtige Angelegenheiten der Wirtschaftspolitik gegen starken Widerstand nicht nur von der Bürokratie sondern auch von Politikern der Regierungspartei durchgesetzt werden. Ein in vielerlei Hinsicht bemerkenswertes Beispiel stellt die Privatisierungspolitik, besonders die Privatisierung der Post dar, die Koizumi als „Zitadelle der Reform“ bezeichnet hat.

Bei der starken politischen Führung von Koizumi spielte die in der Kabinettszentrale eingerichtete „Konferenz für Wirtschaft und das Finanzwesen“ eine entscheidende Rolle. Über wichtige Angelegenheiten der Themenbereiche Wirtschafts- und Finanzpolitik, die früher von mehreren Ministerien und Komissionen in der Regierung abgedeckt worden waren, wurde nunmehr in der Regierung von Koizumi in der „Konferenz“ konzentriert beraten. In dieser „Konferenz“ wurden faktisch Richtlinien der Wirtschafts- und Finanzpolitik, wie z.B. die Richtlinie des Haushaltsplans, beschlossen.

\section{Kritik}

Diese politische Führung der Regierung Koizumi stieß auf gewichtige Kritik. Ein wichtiger Kritikpunkt auch aus der Perspektive der Wissenschaft des Öffentlichen Rechts war, dass die Entscheidungsprozesse unklarer wurden. Es gab in der Tat einige Beispiele, die zeigten, dass Fachleute aus der Wirtschaft, die an den wichtigen Entscheidungen mitwirkten, sich ohne Transparenz sowie Distanz verhielten.

\section{Misserfolge}

Es ist aber vor dem Hintergrund der hier behandelten Fragestellungen noch bemerkenswerter, dass die Regierung Koizumi nach der „Verwaltungsreform“ die einzige Regierung war, die Führungsstärke zeigen konnte. Drei Regierungen mit Premierministern der LDP, die Koizumi nachfolgten, waren von Führungsschwäche gekennzeichnet und können vom Ziel der „Reform“ her als Misserfolg gewertet werden. 
Unter Berücksichtigung der langen Amtzeit von Koizumi ist es schwierig, auf die Frage zu antworten, ob die „Verwaltungsreform“ zur Stärkung der politischen Führung große Wirkungen hatte. Es muß jedoch betont werden, dass die Führungsstärke des Premierministers und seines Kabinetts seit der „Reform“ ständig hinterfragt wurde, während das in den 1970er und 80er Jahren weit seltener der Fall gewesen war.

\section{Bewertung der Verwaltungstätigkeiten}

1. Unabhängige Verwaltungsorganisationen (Benchmarking: Plan-do-check-act cycle)

Die Einführung der Bewertung brachte besonders für die Tätigkeit der unabhängigen Verwaltungsorganisationen große Änderungen. Das gilt auch für die staatlichen Universitäten: Sie wurden 2004 in unabhängige Verwaltungsinstitutionen umgewandelt, weil insgesamt das Personal im Öffentlichen Dienst um 25 Prozent reduziert werden sollte.

Unabhängige Verwaltungsorganisationen müssen nach mittelfristig gesteckten Zielen ihre Aufgaben planmäßig erfüllen. Ihre Leistungen werden von unabhängigen dritten Organen, die aus Sachverständigen zusammengesetzt sind, bewertet. Um diese Bewertung klarer zu machen, sind in den mittelfristigen Plänen der Organisationen konkrete Ziele mit konkreten Zahlen zu nennen, die die Organisationen erfüllen müssen. Dadurch sollte die Tätigkeit der Organisationen verbessert werden. Unnötige Tätigkeiten und Organisationen sollten sogar abgeschafft werden.

\section{Personal- und Sachmittelabbau?}

Die Bewertung der Verwaltungstätigkeit wurde auch in den Ministerien und Behörden eingeführt, war jedoch dort hinsichtlich Wirkung und der Strenge nicht so drastisch wie bei der Betätigung der unabhängigen Verwaltungsorganisationen. Die Anzahl der neuen Anstellung öffentlicher Bediensteter wurde zwar reduziert. Die Finanzausgaben wurden aber nicht so dramatisch verringert, auch nicht unter der Regierung Koizumi. Die Staatsschulden Japans sind daher auch nach der „Reform“ ständig gewachsen.

\section{D. „Erneuerung“ der Verwaltung seit 2009 \\ I. Verlauf der jüngsten Entwicklungen}

Gerade nach dem Rücktritt von Premierminister Koizumi wurde es auch in Japan immer schwieriger, an der politischen Richtung des Neoliberalismus festzuhalten. Für drei Premierminister, die Nachfolger von Koizumi, war es nicht leicht, zwischen der Linie des „erfolgreichen“ Koizumi und der Umgestaltung der politischen Richtlinien klare Akzente zu setzen. Dies wurde als Führungsschwäche gewertet. Die Nachfolger hatten manchmal auch Schwierigkeiten damit, von der Verwaltung verursachte Probleme politisch zu bewältigen. 
Die Demokraten nahmen eine klare Position gegen den Wirtschaftsliberalismus ein. Sie kritisierten auch die Führungsschwäche der LDP und behaupteten die Herstellung des Primats der Politik zu verfolgen. Sie gewannen die Oberhauswahl 2007 und die Unterhauswahl 2009 und ermöglichten somit den Regierungswechsel. Im Jahr 2009 konstituierte sich die Regierung von Premierminister Hatoyama.

\section{Primat der Politik und „Erneuerung “ der Verwaltung}

Eine der wichtigsten Wahlversprechen der Demokraten war die Herstellung des Primats der Politik und die „Erneuerung“ der Verwaltung. Die Regierung Hatoyama traf daher einige Maßnahmen und bereitete dazu notwendige Gesetzentwürfe vor, die auf der Linie der „Verwaltungsreform“ organisatorische Änderungen zur Stärkung der politischen Führung und „Erneuerung“ der Verwaltung vorsahen. Nach dem Fall der Regierung Hatoyama wurde Kan zum nächsten Premierminister ernannt. Er verfolgte in diesem Punkt weiter die Linie von Hatoyama, verlor aber durch die Oberhauswahl in Juli 2010 die Mehrheit im Oberhaus. Viele Gesetzesentwürfe konnten daher vom Parlament nicht verabschiedet werden.

\section{1. „Staatsstrategie“}

Kurz nach dem Regierungswechsel wurde eine Abteilung, die eine stärkere politische Führung ermöglichen sollte, durch Kabinettsbeschluß im Kabinettssekretariat eingerichtet. Diese Abteilung, „Abteilung für die Staatsstrategie“, fällt in die Zuständigkeit eines Staatsministers. Die in der Kabinettszentrale eingerichtete „Konferenz der Wirtschaft und des Finanzwesens“, die stark von der Politik Koizumis geprägt war, spielte während der Regierung der Demokraten keine Rolle mehr.

2. „Konferenz der Erneuerung der Verwaltung“ und populistische Kontrolle für Verwaltungsaufgaben (,Sortierung“)

Die Wahlversprechen vor der Unterhauswahl 2009 enthielten einige Wohltaten in Form einer Abkehr von der Linie des Wirtschaftsliberalismus. Sparmaßnahmen zur Abschaffung der unnötigen Verwaltungstätigkeiten sollten die dazu erforderlichen Finanzquelle erschließen.

Um unnötige Verwaltungstätigkeiten und damit verbundene Finanzausgaben einzusparen, wurde durch Kabinettsbeschluss die „Konferenz zur Erneuerung der Verwaltung“ in der Kabinettszentrale eingerichtet. Sie ist ebenfalls einem Staatsminister zugeordnet und besteht aus dem Premierminister, den betreffenden Staatsministern und vom Premierminister ernannten Sachverständigen.

Die Bewertung der Verwaltungstätigkeiten und Finanzausgaben wurde von der „Konferenz zur Erneuerung der Verwaltung“ auf eine drastische Art und Weise durchgeführt, die als „Sortierung“ bezeichnet wurde. Die Zweckmäßigkeit der von den „Sortierern“ im Vor- 
aus als problematisch angesehenen Tätigkeiten diskutierten die Vollzieher und „Sortierer“ öffentlich. Diese Diskussionen wurden zugleich der Öffentlichkeit zugänglich gemacht, auch live über das Internet. „Sortierer“ bewerteten bei diesen Diskussionen, ob die Tätigkeit effizient und überhaupt erforderlich ist.

Diese öffentliche Bewertung wurde im Zusammenhang mit der Aufstellung des Haushaltsplans 2010 im November 2009 durchgeführt. Durch diese Bewertungen konnten zwar keine ausreichende Finanzquellen, wie vor der Wahl angekündigt, für die versprochenen Wohltaten erschlossen werden. Die „Sortierung“ selbst stieß aber auf ein großes Echo. Sie wurde daher weitergeführt.

\section{Vermehrung der politischen Ämter}

Bei der „Verwaltungsreform“ wurden schon neue politische Ämter sowohl im Kabinettssekretariat als auch in jedem Ministerium geschaffen. Nach den Gesetzesentwürfen sollte die Anzahl der politischen Ämter noch vermehrt werden, wodurch mehr Abgeordnete in der Regierung arbeiten würden.

Nach diesem Plan sollten erstens vorrangig Politiker und nicht Beamte, Entscheidungen treffen; zweitens sollte die politische Führung vorwiegend von der Regierung getragen werden, also nicht vom Parlament oder einer Partei. Ziel war, die im Wahlkampf versprochene Grundlinie der Politik reibungslos durchzusetzen. Die Gesetzesentwürfe dafür konnten zwar aufgrund des Ergebnisses der Oberhauswahl 2010 nicht verabschiedet werden. Die Vereinheitlichung der politischen Führung wurde aber auch von der Regierung Kan häufig betont.

\section{E. Eine Analyse \\ I. Das neue Phänomen: Bürokratie als Problem}

Der Überblick über die Diskussionen und Maßnahmen seit der „Verwaltungsreform“ macht deutlich: Problematisiert wurde die japanische Verwaltung als solche. Die Staatsstruktur in Japan, die von der ,starken“ Verwaltung geprägt war, wurde zwar trotz ihrer Fähigkeit seit langem kritisiert. Die Vorstellung von einer,,überflüssigen und unfähigen Bürokratie“, wie sie in anderen Ländern verbreitet ist, ist in Japan jedoch neu. Die Schwierigkeiten des Staats- und Gesellschaftssystems, das von Einschränkungen und Konventionen beherrscht wird, werden ausschließlich der Verwaltung angelastet.

Hinter dem Konzept, die Verwaltung zu verringern und sie effizienter zu machen, um eine freie und gerechte Gesellschaft zu verwirklichen, stehen aber kaum theoretische Überlegungen darüber, welche Funktionen die Verwaltung in der hochkomplexen modernen Gesellschaft Japans erfüllen soll. Ich habe daher die „Verwaltungsreform“ bei anderer Gelegenheit als ,theoriearm“ bezeichnet ${ }^{9}$.

9

A. Takada (Anm. 1), S. 270. 


\section{Parallelen zwischen „Reform“ und „Erneuerung“}

Der Regierungswechsel von der LDP zu den Demokraten könnte als eine große Wende der Politik angesehen werden. Zwischen der „Reform“ und der „Erneuerung“ lassen sich aber Parallelität und Kontinuität ausmachen.

Bei beiden steht die Stärkung der politischen Führung (,top-down“) im Mittelpunkt. Diese muss sowohl die Effizienz der Verwaltung sichern als auch dem Volk als Herrschaftssubjekt Politik ermöglichen.

Bei beiden kann eine gewisse Parallelität auch darin gesehen werden, dass durch transparente politische Vorgänge harte Maßnahmen gegen die Verwaltung durchgesetzt werden. Insoweit kann die „Sortierung“ im Rahmen der „Erneuerung“ als eine extreme Entwicklungsform der „Konferenz zur Verwaltungsreform“ von Premierminister Hashimoto angesehen werden.

\section{Systemtheoretische Reflexion}

Die starke Kritik gegen die Verwaltung als relativ neue Erscheinung und die Betonung der politischen Führung sowie der Transparenz können mit den gesellschaftlichen Zusammenhängen in Japan erklärt werden.

\section{Informales Verwaltungshandeln}

Die Verwaltung in Japan wurde teilweise durch das informale Verwaltungshandeln, wie z.B. die Verwaltungsanleitung, charakterisiert. Manchmal konnte früher sogar der Eindruck entstehen, das informale Verwaltungshandeln bilde die Regel im Gegensatz zum rechtsgeleiteten Handeln als Ausnahme.

\section{2. „Maximale Mobilisierung der Verwaltungsressourcen“ (Michio Muramatsu)}

Diesen flexiblen Charakter des Verwaltungsstils erklärt ein japanischer Verwaltungswissenschaftler, Michio Muramatsu, mit dem Prinzip der „Maximalen Mobilisierung der Verwaltungsressourcen“. Die Verwaltung in Japan hatte - diesem Ansatz nach - insgesamt relativ wenige Resourcen, sowohl finanzielle als auch personelle. Jeder Beamte und jede Organisation der Verwaltung musste daher auf jeder Ebene flexibel bleiben. Paragraphenreiterei wäre in jedem Fall fatal. Konflikte, die zusätzliche Resourcen benötigen würden, mussten unter diesen Bedingungen dadurch vermieden werden, dass jede Verwaltungsorganisation mit den typischen Verwaltungsadressaten ständig in Kontakt blieb und Vertrauensverhältnisse entwickelte ${ }^{10}$.

Die Entstehung und Entwicklung dieses flexiblen Charakters kann historisch erklärt werden. Der Ausgangpunkt der japanischen Modernisierung war materielle Armut, namentlich Ressourcenarmut. Der Modernisierungsprozess schritt dennoch mit hoher 
Geschwindigkeit voran. Auf dem Weg vom Entwicklungs- zum Industrieland musste die Verwaltung ständig die Initiative ergreifen, damit knappe Resourcen im Zuge der „Institutionalisierung der informellen Institution“ effektiv eingesetzt werden konnten. ${ }^{11}$

Die japanische Gesellschaft ist nun aber eine hochmoderne Gesellschaft mit hoher Komplexität geworden, die allein mit flexiblem Verwaltungshandeln nicht bewältigt werden kann. Ständige Kontakte zu den wichtigen Verwaltungsadressaten können sich zu geschlossenen Beziehungen entwickeln, die unter Umständen bedeutende Interessen Dritter vernachlässigen. Das japanische Staatssystem braucht daher selbst mehr Komplexität und Transparenz.

Für die bisherige Staatsstruktur in Japan kann als charakteristisch bezeichnet werden, dass für Staatorganisationen außerhalb der Verwaltung noch viel Raum für Entwicklung übrig geblieben ist. Die sehr starke Betonung der politischen Führung des Premierministers sowie seines Kabinetts könnte sich daher bis zu einem gewissen Grad auch auf gute Gründe berufen, weil sie zur Erhöhung der Komplexität der Staatorganisationen beiträgt. Auch die Schwerpunktsetzung auf Transparenz kann teilweise als logische Kritik an den geschlossenen Beziehungen der Verwaltung mit den wichtigen Verwaltungsadressaten betrachtet werden.

\section{Notwendigkeit einer systematischen Betrachtungsweise}

Neben dem Premierminister und seinem Kabinett sind aber auch andere Organisationen zu beachten. Zur Stärkung der Funktionen der Gerichte wurden bereits bestimmte Maßnahmen getroffen $^{12}$. Die Rolle des Parlaments soll ebenfalls stärker berücksichtigt werden.

Es genügt jedoch nicht, einzelne Elemente der Staatsorganisationen zu stärken, um die Komplexität der Staatorganisationen zu erhöhen. Jedes Element verbindet sich mit anderen und steht im Verhältnis zu ihnen. Der Gesetzesvorbehalt kann als gutes Beispiel dienen. Das Gesetz unterscheidet zwischen den Angelegenheiten, die im Ermessen der Verwaltung stehen, und jenen, die gerichtlich kontrolliert werden. Je konkreter die Bestimmungen der Gesetze in Japan würden, desto mehr ließe sich die Verwaltung durch Gerichte kontrollieren. Aus systematischer Betrachtungsweise kann die konsequente Entwicklung der Gesetzesvorbehaltslehre in Japan ${ }^{13}$ für die gerichtliche Kontrolle der Verwaltung als notwendig und wichtig angesehen werden.

Zur „Justizreform“ gehören die oben genannten Neuerungen in der Verwaltungsprozessordnung.

Zur Entwicklung des Gesetzesvorbehalts in Japan siehe Bin Takada, Entwicklungstendenzen des Rechtsstaates und der Gesetzmäßigkeit der Verwaltung in Japan aus rechtsvergleichender Sicht, in: Rainer Pitschas/ Shigeo Kisa (Hrsg.), Internationalisierung von Staat und Verfassung im Spiegel des deutschen und japanischen Staats- und Verwaltungsrechts, 2002, S. $145 \mathrm{ff}$. 
4. Primat der politischen Kommunikation?

Aus systemtheoretischer Perspektive kann vom Primat der politischen Kommunikation keine Rede sein. Politische Kommunikation kann in einer Gesellschaft eine wichtige Rolle spielen. Ihre Funktion hat aber Grenzen. Es muss beachtet werden, was sie kann und was nicht, und auch wie sie gut funktionieren kann.

\section{Reformlogik}

Die Besonderheiten der Logik der „Reform“ und der „Erneuerung“ können auch normativ analysiert werden.

\section{1. „Kokumin (Volk, Staatsbürger)“ als Argument}

Der Bericht der „Konferenz zur Verwaltungsreform“ begründet die Notwendigkeit der „Verwaltungsreform“ mit dem „Kokumin“ (Volk; Staatsbürger). Die Logik des Berichts ist nicht so klar, hat aber wahrscheinlich zwei Dimensionen. Erstens kann durch die „Verwaltungsreform“ der „Kokumin“ von Einschränkungen und Konventionen befreit werden. Zweitens kann die Stärkung der Politik durch die „Reform“ die Herrschaftssubjektivität des „Kokumin“ vergrößern.

Diese logische Inkonsequenz folgt nicht nur aus dem unklaren Hintergrund, sondern auch aus der Zweideutigkeit des japanischen Begriffs „Kokumin“. Der Begriff „Kokumin“ meint einerseits den einzelnen Staatsbürger und andererseits das ganzes Volk als Kollektiv. Bei der normtheoretischen Bearbeitung der Staatsstruktur müssen aber die Unterschiede der Dimensionen stärker beachtet werden.

Durch die politische Wende, die zur Trennung vom Wirtschaftsliberalismus führte, könnte in Japan der Aspekt der „Verwaltungsreform“, die Freiheit zu erweitern vielleicht in den Hintergrund rücken. Dies wiederum könnte möglicherweise die Vorstellung einer Identitätsdemokratie erzeugen, die die starke politische Führung des Premierministers und seiner Regierung der Selbstbestimmung des Bürgers direkt zurechnen würde.

2. Kabinett als ein den Volkswillen verwirklichendes Organ?

Abgesehen von der möglichen Vorstellung der Identitätsdemokratie ist es verfassungsrechtlich sehr problematisch, das Kabinett als das den Volkswillen verwirklichende, maßgebliche Staatsorgan anzusehen. Das Organ, das vom Volk direkt gewählt wird und das ganze Volk vertritt, ist nicht das Kabinett, sondern das Parlament (Art. 43 Abs. $1 \mathrm{JV}^{14}$ ). Die Demokratie ist überhaupt, auch in Japan, mehrdimensional, sie hat mehrere Institutionen. Jedes Element sollte in der normativen Theorie der Demokratie einen angemessenen Stellenwert besitzen. Auch bei der Kontrolle der öffentlichen Angelegenheiten hat das (Kokumin) vertreten. 
Kabinett zwar wichtige Funktionen; es ist aber gleichzeitig der wichtigste Gegenstand der parlamentarischen Kontrolle.

\section{Stellung des Parlaments}

Abgesehen davon, dass der Bericht der „Konferenz zur Verwaltungsreform“ die möglichen Maßnahmen der Regierung für die „Verwaltungsreform“ entwarf, hat bemerkenswerterweise das Parlament sowohl bei den „Reformen“ als auch bei den „Erneuerungen“ fast keine Berücksichtigung gefunden. Japan ist eine parlamentarische Demokratie. Das japanische Parlament ist auch zur Kontrolle der vollziehenden Gewalt befugt ${ }^{15}$. Bei der normlogischen Betrachtung der Staatsstruktur muss das Parlament als wesentliches Organ behandelt werden. Die parlamentarische Opposition muss dabei auch als sehr wichtiger Teil berücksichtigt werden.

\section{4. Öffentlichkeit und Transparenz}

Durch die politischen Vorgänge in Japan, die durch transparente Diskussionen die Unterstützung der Bevölkerung zur „Verwaltungsreform“ vergrößerten und Belange der Bürokratie schwächten, lässt sich schwerer zwischen Öffentlichkeit und Transparenz unterscheiden. Für eine rationale und gerechte Verwaltungstätigkeit ist Transparenz wesentlich. Andererseits muss nicht jede Verwaltungstätigkeit Gegenstand öffentlicher Diskussion sein. Bei der Kontrolle der Verwaltung ist die Orientierung an der augenblicklichen Unterstützung der Bevölkerung unangemessen. Die Verwaltungskontrolle hat sicherlich demokratische Elemente. Bloßer Akklamation darf jedoch kein Raum gegeben werden. In diesem Zusammenhang ist die theoretische Unterscheidung zwischen der „öffentlichen Meinung“ und der „Tagesmeinung“ von Hermann Heller ${ }^{16}$ zu beachten.

Die „Reform“ und die „Erneuerung“ hätten sogar unter Umstände die Transparenz gefährdet. Nach der „Reform“ und der „Erneuerung“ sind nun mehr Abgeordnete und Fachleute in der Regierung tätig. Es mag zwar möglich sein, dies demokratisch zu rechtfertigen. Es stellt aber eine dringende Aufgabe dar, darüber nachzudenken, wie die rechtsstaatliche und demokratische Distanz sowie die Transparenz ihrer Tätigkeit dabei bewahrt werden kann.

\section{F. Schluss}

\section{Eigenheiten Japans}

In diesem Beitrag über die organisatorische „Reform“ und die „Erneuerung“ des Verwaltungsrechts wurden die besondere Betonung auf politischer Führung und Transparenz in

Art. 62 JV Jedes der beiden Häuser kann mit Bezug auf die Staatsführung Untersuchungen 16 anstellen und dafür das Erscheinen und die Aussage von Zeugen und Vorlage von Akten fordern.

Hermann Heller, Staatslehre, in: ders., Gesammelte Schriften 3. Band, 1971, S. 277 f. 
Japan als wichtigste Bestandteile hervorgehoben. Es wurde auch versucht, ihre Gründe und ihre Defizite als Reformmaßnahmen vor dem Hintergrund gesellschaftlicher Zusammenhänge der Verwaltung zu erklären. Die Logik von „Reform“ sowie „Erneuerung“ und ihre Defizite werden auch normativ untersucht. Auch dort wurden japanische Eigentümlichkeiten und die Einseitigkeit der Schwerpunktsetzung beobachtet und kritisch analysiert.

Die kritische Beobachtung und Analyse in diesem Beitrag wurden durch die Ereignisse während und nach der Erdbebenkatastrophe in Ostjapan und dem Unfall des Atomkraftwerks in Fukushima 2011 bestätigt.

1) Trotz der Stärkung der politischen Führung konnte die Regierung der Demokraten bei der Bewältigung der Erdbebenkatastrophe und des Unfalls des Atomkraftwerks keine effektiven Maßnahmen treffen. Das zeigt, dass die Stärkung der Spitze der Staatsstruktur (,top-down“) kein Allheilmittel zur Stärkung der konsequenten strategischen Urteils- und Entscheidungskraft der Regierung ist.

2) Nach dem Unfall des Atomkraftswerks in Fukushima wurden wichtige Informationen, die für das Leben und die Gesundheit der Arbeiter sowie der Einwohner sehr bedeutend waren, nicht rechtzeitig veröffentlicht. Die Vernachlässigung und der Zusammenbruch der Sicherheit des Atomkraftwerks wurden überhaupt durch die geschlossenen Beziehungen zwischen der Politik, der Verwaltung, der Industrie, den Medien und der Wissenschaft verursacht. Die Betonung der Transparenz in Japan hatte in Wirklichkeit kaum Wirkungen.

3) Trotz der Erdbebenkatastrophe in Kobe 1995 wurden die Vorbereitungen und die Maßnahmen gegen die nächste Erdbebenkatastrophe nicht gründlich und konsequent durchgeführt. Einige Erscheinungen des Erdbebens 2011 hätten zwar nur schwer vorhergesehen werden können. In der Politik sind aber viele Elemente der Erdbebenkatastrophe, die eigentlich vorher diskutiert werden sollten, politisch nicht thematisiert worden, so dass rechtliche Grundlagen für die Bewältigungen der Katastrophe und des Unfalls auch diesmal oft fehlten. Es ist der japanischen Gesellschaft als Schicksal bestimmt, ständig Erdbebenkatastrophen zu erfahren. In diesem Sinne gibt es in Japan keinen strukturellen Unterschied zwischen dem Erdbeben und dem Taifun. Für das Leben und die Sicherheit der Bevölkerung sind die Vorbereitungen und die Maßnahmen für die nächste Erdbebenkatastrophe wichtig. Sie sollten nicht nur vom Kabinett und der Verwaltung behandelt, sondern besonders im Parlament von Vertretern des ganzen Volkes diskutiert und sogar in Form eines Gesetzes festgelegt werden.

Eigenheiten der „Reform“ und „Erneuerung“ des Verwaltungsrechts in Japan, die durch die kritische Beobachtung und Analyse in diesem Beitrag herausgearbeitet wurden, werden für Wissenschaftler des öffentlichen Rechts in Japan auch weiterhin Gegenstände der kritischen Überlegungen und wissenschaftlichen Bewältigung darstellen. 


\section{Chancen der Rechtsvergleichung}

Solche systematischen und normlogischen Bearbeitungen bieten die Möglichkeit, über universale Rechtserscheinungen wie „Verwaltungsreformen“, bei denen ziemlich ähnliche Einzelphänomene in verschiedenen Ländern beobachtet werden können, konsequente Rechtsvergleichung durchzuführen, um bei der Analyse möglichst wenig den zufälligen Umständen der Beobachtung zu überlassen.

Die systemtheoretischen und normlogischen Erklärungsversuche gegenüber denjenigen, die zu anderen Rechtskreisen gehören und sich stark für Rechtsvergleichung interessieren, bieten gleichzeitig die Möglichkeit, die Rechtserscheinung des eigenen Rechtskreises relativiert zu beobachten und reflektiert neu kennenzulernen. 